\title{
Evaluation of Potential Power Gain with Imputed Genotypes in Genome-Wide Association Studies
}

\author{
Tim Becker $^{\mathrm{a}}$ Antonia Flaquer ${ }^{\mathrm{a}, \mathrm{d}}$ Felix F. Brockschmidt ${ }^{\mathrm{b}, \mathrm{c}}$ Christine Herold ${ }^{\mathrm{a}}$ \\ Michael Steffens ${ }^{\mathrm{a}}$ \\ a Institute for Medical Biometry, Informatics and Epidemiology, ${ }^{b}$ Institute of Human Genetics and \\ 'Department of Genomics, Life and Brain Center, University of Bonn, Bonn, ${ }^{\mathrm{d}}$ Institute of Medical Biometry and \\ Epidemiology, Philipps University Marburg, Marburg, Germany
}

\section{Key Words}

Imputing $\cdot$ In silico genotypes $\cdot E M$-algorithm $\cdot G W A \cdot$ FAMHAP

\begin{abstract}
Background: With the beginning of the era of genome-wide association studies methods to obtain 'in silico' genotypes have gained importance. In this context, an evaluation of genome-wide power levels of current marker panels and the power gain achievable with imputed genotypes are of high interest. Methods: Power for single-marker analysis of imputed genotypes is evaluated via a simulation study based on HapMap data. Power values for genome-wide significance of marker panels of 1,000,000 SNPs are considered for small effect sizes typical of common diseases and large casecontrol samples. In order to evaluate the performance of imputing, we consider a method that is conceptually related to previous approaches. We introduce various modifications which together lead to an alternative implementation of the imputation idea. In particular, a Monte-Carlo (MC) simulation method for association testing of imputed markers is introduced. Results: We show that the incorporation of im-
\end{abstract}

puted genotypes can lead to a substantial power gain for common disease variants if the training sample is large enough. In addition, we show that the MC approach is valuable to for validating association results obtained with imputed genotypes. Discussion: Our simulation study also shows that even denser marker panels than those currently available are needed when sample size is limited. We thus expect that full genome SNP panels will lead to the identification of additional disease variants in the future. Until then, it is desirable that large and ethnically matched training samples genotyped on dense marker panels are available in each country.

Copyright $\odot 2009$ S. Karger AG, Basel

\section{Introduction}

Imputation methods obtain 'in silico' genotypes by combining two sources of information. The first source is a study sample with SNP data for a set $S$ of tightly linked markers. The second source is an additional 'training sample', for instance a HapMap [1] sample, or another study sample. The training set is genotyped for a set $A$ of

\section{KARGER}

Fax +41613061234 E-Mail karger@karger.ch www.karger.com
(C) 2009 S. Karger AG, Basel

0001-5652/09/0681-0023\$26.00/0

Accessible online at:

www.karger.com/hhe
Priv.-Doz. Dr. Tim Becker

Institute for Medical Biometry, Informatics and Epidemiology

University of Bonn, Sigmund-Freud-Str. 25

DE-53105 Bonn (Germany)

Tel. +492282871 4812, Fax+492282871 5854, E-Mail Tim.Becker@ukb.uni-bonn.de 
Table 1. Haplotype distribution with one-to-one correspondence between haplotype and SNP allele

\begin{tabular}{llllll}
\hline Haplotype & $m_{1}$ & $m_{2}$ & $m_{3}$ & $m_{4}$ & $m$ \\
\hline$H_{1}$ & 1 & 1 & 2 & 1 & 2 \\
$H_{2}$ & 1 & 2 & 1 & 2 & 2 \\
$H_{3}$ & 2 & 1 & 1 & 1 & 1 \\
$H_{4}$ & 2 & 1 & 1 & 2 & 2 \\
$H_{5}$ & 2 & 1 & 2 & 1 & 2 \\
$H_{6}$ & 2 & 2 & 1 & 2 & 2 \\
\hline
\end{tabular}

Given the allelic status of $m_{1}-m_{4}$, the allelic status of $m$ can be estimated uniquely for all haplotypes. The $\left\{m_{1}, \ldots, m_{4}\right\}$-haplotype 2-1-1-1 is a one-to-one proxy for allele 1 of marker $m$.

SNP markers with $S \subseteq A$. For a SNP marker $m \in A-S$, genotypes for the study sample can now be estimated by using the haplotype distributions of the marker sets $A$ and $S$. For the study sample, there is full information on the $S$-haplotypes (the haplotypes referring to $S$ ). Under the assumption that study sample and training sample belong to ethnically closely related populations, the haplotype distribution of $S$ and the restriction of the haplotype distribution of $A$ to $S$ will be comparable. Vice versa, it will often be possible to predict the allelic status of the marker $\mathrm{m}$ of a certain individual given its $S$-haplotypes and given the haplotype distribution of $A$. Evans et al. [2] used this principle to predict the most likely genotypes of individuals. They showed that prediction works extremely well in regions of high linkage disequilibrium (LD). De Bakker et al. [3] transfer this idea to association testing and consider the situation were one allele of SNP $m$ lies on exactly one $(S \cup m)$-haplotpye. In this case, the 1 degree of freedom (d.f.) association test for haplotype $h=$ $\left.H\right|_{S}$ is closely related to an association test for SNP $m$. In case that the haplotype distribution of the study sample is not richer than that one of the training sample and if there are no phase-ambiguities, single-marker test and haplotype test are equivalent. A respective example can be found in table 1 which is an adaptation of table 1 in [4].

The ideas of de Bakker et al. [3] have been extended in a paper by Nicolae [4]. In that paper, every marker $m$ is tested for association, even if there is no one-to-one correspondence of a marker allele with a single haplotype. Consider the haplotype distribution of table 2. Allele 2 of marker $\mathrm{m}$ is now located on two different haplotypes. Thus, the scenario is not covered by the approach of de Bakker et al. [3]. The fact that allele 2 is located on
Table 2. Hypothetical haplotype distribution of training sample

\begin{tabular}{llllll}
\hline Haplotype & $m_{1}$ & $m_{2}$ & $m_{3}$ & $m$ & frequency \\
\hline$H_{1}$ & 1 & 1 & 1 & 1 & 0.11 \\
$H_{2}$ & 1 & 1 & 2 & 2 & 0.04 \\
$H_{3}$ & 1 & 2 & 1 & 1 & 0.30 \\
$H_{4}$ & 2 & 1 & 1 & 1 & 0.01 \\
$H_{5}$ & 2 & 1 & 2 & 1 & 0.19 \\
$H_{6}$ & 2 & 1 & 2 & 2 & 0.35 \\
\hline
\end{tabular}

Haplotypes $H_{5}$ and $H_{6}$ lead to identical restrictions with respect to the marker set $\left\{m_{1}, \ldots, m_{3}\right\}$. Therefore, unique imputation of $m$ is not possible for individuals with at least one copy of haplotype $h=\left.H_{5}\right|_{\left\{\mathrm{m}_{1}, \ldots, \mathrm{m}_{3}\right\}}=\left.H_{6}\right|_{\left\{\mathrm{m}_{1}, \ldots, \mathrm{m}_{3}\right\}}$.

different haplotypes is, however, not the reason that unique imputation is not possible. The point is that the restrictions of haplotypes $H_{5}$ and $H_{6}$ with respect to the marker set $\left\{m_{1}, \ldots, m_{3}\right\}$ are identical, $H_{5} \mid\left\{m_{1}, \ldots, m_{3}\right\}=$ $H_{6} \mid\left\{m_{1}, \ldots, m_{3}\right\}=h$. Probabilistic imputation of $m$ is yet possible. Based on the frequencies of $H_{5}$ and $H_{6}$ in the training data set, haplotype $h$ will be extended by allele 1 with probability $0.19 /(0.19+0.35)$, and extended with allele 2 with probability $0.35 /(0.19+0.35)$. With this principle it is possible to give allele and genotype probabilities for all individuals of the study sample. Summation of the probabilities according to allelic status and disease status leads to a proxy of the frequency distribution $U_{1}$ of allele 1 of SNP $m$ in the study sample. Nicolae [4] describes how an asymptotic single marker test for $\mathrm{m}$ can be constructed.

Marchini et al. [5] developed the ideas into a modelbased imputation method. Genotype probabilities are formulated in terms of parameters of a Hidden Markov model. The imputation method uses information from all neighbouring SNPs, but 'in a way that decreases with genetic distance' [5], where genetic distance is measured via local recombination rates obtained from the HapMap. $\mathrm{p}$ values for association testing are obtained with the help of Bayes factors that account for conducting multiple, correlated tests. In their power study, the authors observe a power gain particularly for rare disease SNPs that are poorly tagged $\left(r^{2}<0.8\right.$ for all genotyped SNPs). While the approach by Nicolae [4] has a problem when for a given $S$-haplotype $h$ there is no $(S \cup\{m\})$-haplotype $H$ in the training sample with $h=\left.H\right|_{S}$, an elegant solution for this problem naturally occurs within the population genetics framework by Marchini et al. [5]. 
A further alternative has been given by Browning and Browning [6]. They introduce a fast haplotype phasing algorithm that is based on localized haplotype clustering. The authors evaluate computer running time for haplotype phasing and the accuracy of allelic imputation, but power issues related to imputed SNPs are not considered. Concerning imputation accuracy and speed, their algorithm turns out to be suitable for application to genomewide association studies (GWAS).

In addition to imputing methods for unrelated individuals, it is straightforward to use pedigree data for imputation of genotypes. A respective method has been described by Burdick et al. [7]. The authors show that a small training sample is sufficient to obtain information on untyped genotypes in a much larger set.

The scenario considered de Bakker et al. [3] shows that association methods are closely related to haplotype tests. This observation is generalized in the weighted haplotype method described by Zaitlen et al. [8]. In this paper we will, however, restrict ourselves to the notion of imputing a single marker and try to improve the respective association test. As becomes apparent from the paper by Browning and Browning [6], the choice of the algorithm for haplotype inference is crucial for allelic imputation. In the mid of the 90ties, maximum-likelihood (ML) frequency estimation with the expectation-maximization(EM-)algorithm became a standard [9-11]. The EM-algorithm has then been criticized since its running time increases strongly with the number of markers considered. The partition-ligation technique by Qin et al. [12] overcomes this limitation, but presumably at the price of accuracy [13]. As a consequence, alternative not EM-based approaches have become popular, as for instance, implemented in PHASE [14] and later on fastPHASE [15]. The PHASE algorithm is based on coalescent theory and makes use of haplotype similarity within a MarkovChain framework. While the not-EM based approaches can handle much larger number of markers (up to 1000 SNPs), their running time increases with the number of individuals. As has been observed by Browning and Browning [6], it has become common to compare the performance of haplotype phasing algorithms on small samples $[12,14,16]$. Such practice might lead to underestimation of the performance of the classic ML/EM methods which by design depend on the availability of not too small samples. We are going to show here that the classic EM-algorithm, as implemented in FAMHAP [17], leads to haplotyping quality that translates into power gain via imputed genotypes.

Association Analysis with EM-Imputed Genotypes

\section{Methods}

The imputing method we introduce in this work is conceptually related to previous approaches. There are, however, various modifications which taken together lead to a method and implementation in its own right. We summarize the key features of our approach as follows:

\section{Haplotype Determination in Joint Data Set Consisting of}

Training Sample and Study Sample

Trios are known to improve accuracy and speed of haplotype frequency estimation $[7,16,18,19]$. Therefore, it is obvious to use HapMap trio samples as training samples for case-control study samples. By considering trios and unrelated individuals simultaneously, haplotype phasing and imputing accuracy is improved.

\section{Usage of Many Prediction SNPs for Imputation}

The progressive-extension technique (David Clayton, SNPHAP, unpublished) that is implemented in FAMHAP allows to determine haplotypes of many SNPs and to use them for imputation. From a formal point of view, the best solution is to always choose the set used for prediction as large as possible. Indeed, if haplotype phasing and transferability of the haplotype distribution from the training sample to the study sample are assumed to be perfect, for any set $S_{1}$ with $S \subseteq S_{1}$ the prediction of $m$ can only improve compared to that achievable using $S$ : if a $S$-haplotype predicts $m$, so will any $S_{1}$-haplotype $H$ with $\left.H\right|_{S_{1}}=\left.H\right|_{S}$. On the other hand, if a $S$-haplotype does not allow prediction of $m$, it is still possible that there are multiple $S_{1}$-haplotypes with $\left.H\right|_{S_{1}}=\left.H\right|_{S}$ which all allow unique prediction. Therefore, it is recommendable to use all SNPs from the LD region for prediction. There is, of course, a practical limitation. For instance, using SNPs in weak or no LD with the SNP will not improve imputing but will lead to increased computer running time.

\section{Usage of Genotype Prediction Score}

Even if a $S$-haplotype extends uniquely to a $S \cup\{m\}$-haplotype it is possible that there are genotypes that can not be estimated uniquely because of phase uncertainty. To see this, let $h_{1}$ and $h_{2}$ be $S$-haplotypes which extend uniquely to allele 1 of SNP $m$ and let $h_{3}$ and $h_{4}$ be $S$-haplotypes which extend uniquely to allele 2 of SNP $m$. Although extension is unique, the genotype at $m$ is not unique if there is a unphased multi-marker genotype at $S$ with possible haplotype explanations $\left(h_{1}, h_{2}\right)$ and $\left(h_{3}, h_{4}\right)$. The genotype at $m$ will be predicted to be $(1,1)$ or $(2,2)$ with the conditional likelihood weights of the respective haplotype explanations. The example demonstrates that a general prediction measure for a SNP $m$ and a prediction set $S$ (a generalized $r^{2}$, for instance,) will not always be sufficient. Instead, an additional measure for each imputed genotype can be useful. Marchini et al. [5] remove all genotypes with a prediction probability of less than 0.95 from subsequent association analysis. We thought that this criterion could be improved by taking the allele frequency of the imputed marker $m$ into account. For example, if the minor allele frequency $f$ of allele 1 is equal to 0.01 , the a priori probability that an unknown genotype is equal to $(2,2)$ is $0.99^{2}=0.98$. Thus, an imputing probability of 0.95 is not a sign of good prediction in this case. In order to formalize these thoughts, let $f_{i j}$ be the probability of genotype $(i, j), 1 \leq i \leq j \leq 2$, assuming Hardy-Weinberg-equilibrium and minor allele frequency $f$ (derived from the joint study and training 
sample). Let $p_{i j}$ the prediction probability obtained by imputation of genotype $(i, j)$ for a given individual. After some experimentation we defined the genotype imputation score $g p s$ as $g p s$ : = $\left(s_{11}+s_{12}+s_{22}\right) / 3$ with $s_{i j}=\left(p_{i j}-f_{i j}\right) /\left(1-f_{i j}\right)$, if $p_{i j} \geq f_{i j}$, and $s_{i j}=$ $\left(f_{i j}-p_{i j}\right) /\left(f_{i j}\right)$ else. The $s_{i j}$ are the difference between observed and expected genotype probability, divided by the maximally possible difference between the two values $p_{i j}$ and $f_{i j}$. For instance, if $p_{i j} \geq$ $f_{i j}$, the maximal difference is $1-f_{i j}$. Note that $g p s=1$ if and only if the genotype can be imputed uniquely and that $g p s=0$ if and only if $f_{i j}=p_{i j}$ for all $(i, j)$, i.e., in case the haplotype distribution does not yield additional information. Thus, $g p s$ is equal to zero if and only if the $S$-haplotypes and the SNP to be imputed are in linkage equilibrium and no information on association with the phenotype can be gained. An example for the usage of the score is given in the section 'Application to Real Data Set'.

4. Usage of Monte-Carlo Simulations for Association Testing

The most important novelty is that uncertainty of imputed genotypes is accounted for via a Monte-Carlo simulation approach (see below). With this technique, we first obtain robustness against non-fitting haplotype distributions of training and study sample and, second, do not have to rely on asymptotic distributions.

\section{Algorithm}

We describe the algorithm for a study case-control sample that is genotyped for a set S of SNPs in LD and a reference (HapMap) trio sample that is genotyped for $S$ and one additional SNP $m$. Note that the whole formulation works for a case-control sample as training sample, as well. However, we explicitly do not recommend to merge study samples genotyped for different marker panels. Instead, we require imputation in both cases and controls through an independent training sample whose family units are not directly used for association testing.

An association test for SNP $m$ in the case-control sample is constructed as follows:

1. ML-frequency estimates for the $S \cup\{m\}$-haplotypes are computed from the joint sample of cases, controls and trios. For this purpose, the progressive-extension of the EM-algorithm as implemented in FAMHAP is used. The extension starts with the SNPs from set $S$. SNP $m$ is introduced at last. In this way, speed is optimized since SNP $m$ is genotyped only for the trios.

2. Next, each haplotype explanation of each individual from the case-control sample is assigned a conditional likelihood weight. Let $k, l$ be haplotypes and let $h_{k}, h_{l}$ be the corresponding ML-frequencies obtained as described in step 1 . Let $C_{i}$ be the set of all possible haplotype explanations of a given individual $i$. Finally, define $\gamma_{k l}=2$, if $\mathrm{k} \neq l$, and $\gamma_{k l}=1$, else. The conditional likelihood weight $w_{k, l}$ of a haplotype explanation $(k, l)$ of individual $i$ can be computed as

$$
w_{k, l}=\frac{\gamma_{k l} h_{k} h_{l}}{\sum_{(K, L) \in C_{i}} \gamma_{K L} h_{K} h_{L}} .
$$

3. Each $S \cup\{m\}$-haplotype $h$ contains information on allelic status at SNP $m$. Let 1, 2 be the alleles of SNP $m$. For a haplotype explanation $(k, l)$, we define $\beta=\beta(k, l)$ as $\beta=2$, if both $k$ and $l$ carry allele 1 at SNP $m, \beta=0$, if both $k$ and $l$ carry allele 2 at SNP $m$, and $\beta=1$, else. In other words, $\beta$ counts the number of occurrences of allele 1 of SNP $m$ in the haplotype explanation $(k, l)$.

4. For each individual, the genotype prediction probabilities $p_{i j}, 1 \leq i \leq j \leq 2$, are computed as

$$
\begin{aligned}
& p_{11}=\sum_{(K, L) \in C_{i} ; \beta(K, L)=2} w_{K, L} \\
& p_{12}=\sum_{(K, L) \in C_{i} ; \beta(K, L)=1} w_{K, L}
\end{aligned}
$$

and

$$
p_{22}=\sum_{(K, L) \in C_{i} ; \beta(K, L)=0} w_{K, L}
$$

5. (Optional) Using the a priori genotype probabilities $f_{i j}, 1 \leq$ $i \leq j \leq 2$, the genotype prediction score $g p s$ can be computed for each individual. Individuals with $g p s$ below a pre-defined threshold can be excluded from analysis.

6 . By summing $2 p_{11}+p_{12}$ over all remaining affected individuals, we obtain the total count of allele 1 of SNP $m$ in cases. Analogously, we obtain allele counts in controls, and allele counts for allele 2 in cases and controls. From the resulting $2 \times 2$ contingency table, we compute the standard test statistic

$$
T_{0}=\frac{(a d-b c)^{2} \times(a+b+c+d)}{(a+b)(c+d)(a+c)(b+d)} .
$$

Here, $a, b, c, d$ denote the four different cell counts.

7. A p value is obtained with Monte-Carlo simulations. In each permutation replicate of the simulations, we randomly permute affection status as previously described by Becker et al. [20]. The set of weighted haplotype explanations and hence the imputed genotype of each individual remains unchanged in the simulated data sets. As usual, the $\mathrm{p}$ value is computed as

$$
\frac{\left|\left\{i: T_{i} \geq T_{0}\right\}\right|}{\mathrm{n}} \text {. }
$$

$\mathrm{n}$

Here, $n$ is the number of permutation replicates and $T_{i}$ is the value of the test statistic in permutation replicate $i$.

The MC approach shows that our association test for imputed genotypes is formally equivalent to a haplotype test defined by dividing the different haplotypes into two classes according to allelic status at $m$. Since the subdivision is defined by the SNP that shall be imputed, we can speak of a single-marker test for the imputed SNP.

It has been shown by Nicolae [4] that the test statistic $T_{0}$ considered above does not follow $\chi^{2}$ distribution with 1 degree of freedom (d.f.). Therefore, we use the MC approach to obtain correct $\mathrm{p}$ values. The $\mathrm{MC}$ approach has the advantage that it is not necessary to rely on the asymptotic distribution that has been introduced by Nicolae [4]. We emphasize, however, that we do not know how the test by Nicolae behaves with data from the extreme tail of the distribution.

A drawback of the MC approach is running time. In order to compute $\mathrm{p}$ values with a precision that confirms genome-wide significance, a huge amount $\left(>10^{8}\right)$ of permutation replicates is needed. Real data analysis of selected SNPs is feasible with our implementation, but a power simulation study cannot be carried out with the MC approach. We will evaluate parts of our power study by computing $p$ values under the assumption that $T$ is $\chi^{2}$ - 
distributed with 1 d.f. This procedure can serve as a pre-test in real data analysis. We will show that the pre-test does not lead to inflated type I error, but rather to a conservative test. We thus pay the price that we might slightly underestimate the power of imputing in our simulation study. In order to investigate the performance of the pre-test, we will apply it also to the situation when certain individuals are removed from data analysis due to low genotype imputation score.

\section{Simulation Study}

The goal of our simulation study was to simulate data that mimics a real data analysis situation. We followed the idea of the set-up introduced by de Bakker et al. [3]. In brief, the idea is to 'select' a particular SNP to be disease associated and derive the haplotype distribution of all SNPs that exist in its vicinity. Based on this haplotype distribution and the allelic status at the disease marker, a case-control sample is simulated. In order to match the number of SNPs that are available in real data analysis, information on certain SNPs in the neighborhood is ignored and not used for power evaluation.

In this paper, we used HapMap data of the ENCODE 9 region [The International HapMap Consortium, 2003] for which 48 unrelated individuals were sequenced. All identified SNPs were subsequently genotyped in the HapMap trio samples. Here, we consider the CEU population (30 trios). For this 'complete' marker panel, we considered windows of 62 non-monomorphic SNPs. The number 62 was chosen since it borders the current limit of allowed SNPs in FAMHAP and, since it leads to windows sizes for which it is reasonable to assume that the SNPs are in LD. The haplotype frequency distribution of the windows was estimated with FAMHAP. Within each window, SNP 32 was assigned to be the disease marker. A multiplicative disease model was assumed. Based on the haplotype distribution and the allelic status at the disease marker, samples of 2,500 cases and 2,500 controls were simulated. The population frequency of the disease allele was set to be equal to the frequency in the ENCODE data. Only disease markers with disease allele frequency between 0.05 and 0.95 were considered, i.e., we focused on rather common disease variants. Depending on this allele frequency, penetrance was chosen such that a genome-wide power level (see the results section for a definition of 'genome-wide power') of about $80 \%$ should achieved for the 1 d.f. single-marker test of full genome 'real' SNP data. For this purpose, we conducted a small pilot study to determine the penetrances. We assumed the penetrance of the wildtype genotype to be either 0.01 or 0.10 , with equal probability. We note that the assumed allelic risk ratios were rather small (between 1.27 and 1.5 , depending on allele frequency) and typical of recent findings. The $80 \%$ power level is achieved by the large sample size. We also simulated data sets under the null hypothesis by choosing equal penetrance for all genotypes of the 'disease' SNP. The marker windows were moved over the ENCODE 9 region with step size 1 , but windows with rare disease variants were ignored. When the end was reached, we started again from the beginning of the region. We stopped our simulation study after 3000 marker windows had been analyzed.

From the complete SNP data of each window, additional data sets with a selected set of SNPs were produced by setting genotype data of non-selected SNPs to zero. The SNP-reduced data sets were produced in such a way that they matched either the density of the HapMap reference panel or marker panels with 1,000,000 SNPs. Our aim was, thus, to evaluate power of marker panels that will be available in the near future. According to the latest publication of The International HapMap Consortium [21], the HapMap provides genotype data for about 2,600,000 quality controlled SNPs for the CEU panel. Furthermore, about 9-10 million common SNPs are estimated to be present in the assembled human genome [21]. For our simulation study we assumed that the total number of SNPs in the reference genome is 4,000,000. This is a reasonable reduction since, firstly, the CEU population will be less diverse than the whole population, and, secondly, the ENCODE reference panel will fail to contain a lot of SNPs because of its limited sample size. According to these assumptions, our results will refer to marker panels that cover $1,000,000 / 4,000,000=$ $25 \%$ and to a (HapMap) reference panel that covers 2,600,000/ $4,000,000=65 \%$ of all SNPs in the CEU population. Thus, if there are actually more than $4,000,000$ SNPs int the CEU population, the true power of the marker panels we consider will actually be lower than estimated here. However, the additional SNPs will be in LD with the markers considered here, such that this bias will not be too large.

According to the percentages computed above and with a marker window size of 62 SNPs, each marker window contains 41 randomly selected (Pseudo-)HapMap SNPs. The 1,000,000 marker $(1 \mathrm{M})$ panels correspond to 16 SNPs per 62-marker window. The $1 \mathrm{M}$ panels were selected in two different ways. In order to mimic the idea of the Illumina ${ }^{\circledR}$ panels, 16 out of 41 HapMap SNPs were selected based on $r^{2}$ values with the (Pseudo-)HapMap markers. In order to mimic Affymetrix ${ }^{\circledR}$ panels, 16 out of 62 SNPs were selected at random. In the following, we will refer to these panels as $1 \mathrm{M} \_$TAG and 1M_RANDOM. We explicitly note that we did not use actually available commercial panels. Data for the training sample was obtained in two different ways:

\section{Small Training Sample (STS)}

Our simulation set-up assumes that the HapMap haplotype distribution is the true population distribution. In practice, only a small HapMap training sample is available. Therefore, we sampled for each marker window a hypothetical HapMap training sample by randomly choosing 30 trios according to the 'true' haplotype distribution. The simulated training sample shows a much less diverse haplotype distribution than the 'true' population, because sample size of the training sample is small. In this way, we mimic reality, because for real data it is likely that the small HapMap sample has a less diverse haplotype distribution.

\section{Ideal Training Sample (ITS)}

Here, we added to each data set the respective HapMap trio data as training sample. Thus, the full diversity of the study sample will be found in the training sample. In this sense, this simulation set-up is ideal. In practice, such a situation can nearly be reached with a ethnically matching training sample that is large enough to capture the complete diversity.

Under both scenarios, we also considered full sequence training data (trio data set for all 62 SNPs) to evaluate the potential of a complete reference panel. 


\section{Results}

\section{Simulations under the Null Hypothesis}

We simulated 2000 different SNP windows under the null hypothesis. We considered both the $1 M_{-}$TAG and the 1M_RANDOM panel together with ideal training sample. For both situations, we considered only those windows for which SNP 32 was in the HapMap panel, but not in the marker panel. Within each window, SNP 32 was then imputed using all SNPs that were both in the marker panel (genotyped in study sample) and the HapMap reference panel (genotyped in the training sample). Note that not all markers from the 1M_RANDOM panel are in the reference panel. $p$ values were determined either with the MC approach (using 999 permutation replicates for each $p$ value computation) or using the $\chi^{2}$ distribution (pre-test). For the pre-test, we considered for different $g p s$-thresholds $(\{0.00,0.50,0.80,1.00\})$ and discarded individuals below the threshold from association testing.

By design, the MC approach leads to a test with proper level. Indeed, haplotype estimation is carried out just once under the null hypothesis, by considering the joint case-control and training sample. In particular, the validity of the test should not depend on the SNPs selected for prediction. Even if those were not in LD, the MC-procedure would guarantee a valid test. This expectation is confirmed by the results of the simulation study. Table 3 contains empirical significance levels for a nominal level of $\alpha=0.05$. We do not observe significant deviations from the nominal level.

Using the $\chi^{2}$ approximation rather than simulations for $\mathrm{p}$ value computation leads to a conservative test, in particular when all individuals are kept ( $g p s$ threshold equal to zero). This result can easily be explained. When imputation is not perfect, individuals get prediction probabilities different from zero for all three genotypes. In other words, individuals with poorly imputed genotype will add noise to the contingency table. As a consequence, the usual test statistic $T$ for $2 \times 2$ is not $\chi^{2}$-distributed. In concordance with this contemplation, conservativeness is less strong with higher thresholds for gps. Already with a $g p s$ threshold of 0.50 the degree of conservativeness is small and when only uniquely imputed individuals are considered ( $g p s$ threshold 1.00), no deviation from the nominal 0.05 level can be observed.

\section{Point-Wise Power Comparison}

For power comparisons related to unadjusted $\mathrm{p}$ values, we considered both the 1M_TAG and the 1M_RANDOM
Table 3. Empirical significance levels $(a=0.05)$

\begin{tabular}{llll}
\hline Marker panel & $g p$ s threshold & MC & pre-test $\left(\chi^{2}\right)$ \\
\hline 1M_RANDOM & 0.00 & 0.047 & 0.021 \\
& 0.50 & & 0.033 \\
& 0.80 & & 0.039 \\
& 1.00 & & 0.049 \\
\hline 1M_TAG & 0.00 & 0.052 & 0.020 \\
& 0.50 & & 0.041 \\
& 0.80 & & 0.046 \\
& 1.00 & & 0.053 \\
\hline
\end{tabular}

panel. In addition to imputation on a HapMap scale, we also considered imputation using all SNPs from the marker window in order to evaluate the potential of a full sequence reference panel. Under all constellations of marker and reference panel, we considered only those marker windows for which the disease SNP 32 was not in the marker panel, but in the reference panel. SNP 32 was then imputed using all SNPs that were both in the marker panel (genotyped in study sample) and the reference panel (genotyped in training sample). We considered power of the MC approach and power of the pre-test for four different $g p s$-thresholds $(\{0.00,0.50,0.80,1.00\})$. According to a recent publication of the Wellcome Trust Case Control Consortium [22], a p value of $\alpha=5 \times 10^{-7}$ is regarded to be sufficient to assure genome-wide significance. We wanted to address the following question: Given a genotyped marker reaches that level, how likely is it that the cut-off is reached when the SNP can only be imputed? Therefore, we considered only marker windows for which the genotyped SNP 32 would have reached the $5 \times 10^{-7}$ level. We note that this was the case for about $90 \%$ of the marker windows we simulated. (Remember that the data was simulated such that adjusted genomewide power was $80 \%$ ). We computed the portion of those marker windows for which the imputed SNP 32 had a $p$ value below $5 \times 10^{-7}$. For simplicity, we will refer to this portion as 'power', although it would be more precise to speak of conditional power. In addition, we computed the portion of windows for which at least one of the genotyped SNPs of the window reached that level of significance. Finally, we calculated those portions also for a level of $5 \times 10^{-4}$, in order to see how often the window would at least reach a reasonable level for selection for a follow-up study.

In order to compute the power of the MC test, for each data set, we first computed the $\mathrm{p}$ value of the pre-test ( $g p s$ 
Table 4. Point-wise power values ${ }^{\mathrm{a}}$

\begin{tabular}{|c|c|c|c|c|c|c|c|c|}
\hline \multirow[t]{2}{*}{ Marker panel } & \multirow{2}{*}{$\begin{array}{l}\text { Reference } \\
\text { panel }\end{array}$} & \multirow{2}{*}{$\begin{array}{l}\text { gps } \\
\text { threshold }\end{array}$} & \multicolumn{3}{|l|}{$\alpha=5 \times 10^{-7}$} & \multicolumn{3}{|c|}{$\alpha=5 \times 10^{-4}$} \\
\hline & & & $\begin{array}{l}\text { imputed } \\
\text { D-SNP ITS }\end{array}$ & $\begin{array}{l}\text { imputed } \\
\text { D-SNP STS }\end{array}$ & $\begin{array}{l}\text { best other } \\
\text { SNP }\end{array}$ & $\begin{array}{l}\text { imputed } \\
\text { D-SNP ITS }\end{array}$ & $\begin{array}{l}\text { imputed } \\
\text { D-SNP STS }\end{array}$ & $\begin{array}{l}\text { best other } \\
\text { SNP }\end{array}$ \\
\hline \multirow{11}{*}{$\begin{array}{l}\text { 1M_RANDOM } \\
\text { 1M_RANDOM }\end{array}$} & \multirow{6}{*}{$\mathrm{HM}^{\mathrm{d}}$} & - & - & - & \multirow[t]{11}{*}{0.514} & - & - & \multirow{11}{*}{0.746} \\
\hline & & 0.00 & 0.440 & 0.372 & & 0.574 & 0.546 & \\
\hline & & $0.00 \mathrm{MC}$ & 0.451 & 0.393 & & 0.615 & 0.579 & \\
\hline & & 0.50 & 0.463 & 0.383 & & 0.603 & 0.551 & \\
\hline & & 0.80 & 0.417 & 0.350 & & 0.534 & 0.521 & \\
\hline & & 1.00 & 0.348 & 0.308 & & 0.437 & 0.441 & \\
\hline & \multirow[t]{5}{*}{$\mathrm{FS}^{\mathrm{e}}$} & 0.00 & 0.601 & 0.484 & & 0.795 & 0.761 & \\
\hline & & $0.00 \mathrm{MC}$ & 0.663 & 0.565 & & 0.832 & 0.801 & \\
\hline & & 0.50 & 0.615 & 0.545 & & 0.821 & 0.786 & \\
\hline & & 0.80 & 0.590 & 0.525 & & 0.839 & 0.721 & \\
\hline & & 1.00 & 0.520 & 0.471 & & 0.811 & 0.688 & \\
\hline 1M_TAG & \multirow{5}{*}{ HM } & - & - & - & \multirow[t]{11}{*}{0.568} & - & - & \multirow[t]{11}{*}{0.822} \\
\hline \multirow{10}{*}{ 1M_TAG } & & 0.00 & 0.693 & 0.487 & & 0.871 & 0.744 & \\
\hline & & $0.00 \mathrm{MC}$ & 0.735 & 0.577 & & 0.896 & 0.785 & \\
\hline & & 0.50 & 0.726 & 0.569 & & 0.871 & 0.772 & \\
\hline & & 0.80 & 0.693 & 0.529 & & 0.837 & 0.752 & \\
\hline & \multirow{6}{*}{ FS } & 1.00 & 0.643 & 0.479 & & 0.787 & 0.704 & \\
\hline & & 0.00 & 0.710 & 0.508 & & 0.888 & 0.850 & \\
\hline & & $0.00 \mathrm{MC}$ & 0.746 & 0.574 & & 0.912 & 0.881 & \\
\hline & & 0.50 & 0.738 & 0.565 & & 0.892 & 0.871 & \\
\hline & & 0.80 & 0.666 & 0.535 & & 0.856 & 0.861 & \\
\hline & & 1.00 & 0.598 & 0.510 & & 0.804 & 0.825 & \\
\hline
\end{tabular}

${ }^{\mathrm{a}}$ Portion of windows that reach the $\alpha$ level; ${ }^{\mathrm{b}}$ power for ideal training sample; ${ }^{\mathrm{c}}$ power for small training sample; ${ }^{\mathrm{d}}$ imputation of HapMap data; ${ }^{\mathrm{e}}$ imputation of full sequence data.

threshold 0.00$)$. In case this p value lay in of the interval $\left[5 \times 10^{-3} ; 5 \times 10^{-8}\right]$, we recomputed the $p$ value with the $\mathrm{MC}$ approach, otherwise we relied on the pre-test $\mathrm{p}$ value to judge whether the MC $\mathrm{p}$ value lay above or below the thresholds $5 \times 10^{-4}$ or $5 \times 10^{-7}$. In this way, the simulation study was kept computationally feasible. We note that the MC $\mathrm{p}$ values were smaller than the pre-test $\mathrm{p}$ values for $90 \%$ of the data sets (not surprisingly). None of the pre-test $\mathrm{p}$ values was lower by a factor of 10 or more than the $\mathrm{MC}$ p value. Our power estimates of the $\mathrm{MC}$ approach should, therefore, not, be biased by the approximate computation.

The results of the point-wise power comparison are shown in table 4 . The rows 'Best Other SNP' contain the percentage of windows for which one of the genotyped SNPs reached the significance level.

We first discuss the results obtained with the ideal training sample (ITS). With the 1M_RANDOM panel, in $51.4 \%$ of all cases the $5 \times 10^{-7}$ level was reached by a last one genotyped SNP. The respective number for the $1 \mathrm{M}_{-}$
TAG panel was somewhat higher (56.8\%). Thus, the tagging approach leads to increased point-wise power. The numbers show that for a large portion of windows that do not contain the disease SNP, none of the genotyped SNPs reaches the $5 \times 10^{-7}$ level. Thus, many true associations will be lost due to incomplete SNP coverage even with a $1 \mathrm{M}$ SNP chip. The situation is, however, not actually that bad. We also observe that for a high portion of windows (1M_RANDOM: 74.6\%; 1M_TAG: $82.2 \%$ ) at least a suggestive significance level of $5 \times 10^{-4}$ is reached. We also note that these values, of course, depend on the assumed allelic effects. If the genome-wide power level of the simulation study would have been chosen higher than $80 \%$, the (conditional) power $\mathrm{p}$ values just discussed would have been higher, as well. In addition, the high portion of significant findings at the moderate threshold also indicates that large sample size will make up for power lost due to incomplete coverage.

The column 'Imputed D-SNP' contains the portion of windows for which the imputed disease SNP reaches the 
significance level. We first consider $\alpha=5 \times 10^{-7}$ and the MC $p$ values. We observe that for the 1M_TAG panel the power levels are much better than for the best other SNP. For instance, with the HapMap reference panel, the power level improves from 56.8 to $73.5 \%$ with the MC p values. This shows that imputing actually makes sense. Not surprisingly, power improves further when full sequence training data is available. For the 1M_RANDOM panel and HapMap reference panel, power with imputed SNP $(45.1 \%)$ is lower than for the best other SNP $(51.4 \%)$. This is easily explained: many of markers from the 1M_RANDOM panel are not part of the HapMap reference panel. Therefore, imputing is based on fewer markers and no power gain can be observed. The power $\mathrm{p}$ value for the full sequence training data confirms this interpretation. Here, the power of the imputed SNP improves to $66.3 \%$, a gain that is comparable to that reached with the $1 \mathrm{M}_{-}$ TAG panel.

The power level of the pre-test is always lower than the $\mathrm{MC}$ power level. In particular, the pre-test with gps threshold of 1.00 has markedly reduced power under all constellations, although it is not conservative. We conclude that it does not make sense to reduce association testing to those genotypes which can be imputed with $100 \%$ certainty. Otherwise, there is no clear trend for the power levels of the other $g p s$ thresholds. The 0.50 threshold performs the best on average, but not under all scenarios. Thus, a combined pre-test/MC-approach with $g p s$ threshold equal to 0.50 for the pre-test turns out to be the strategy of choice.

With the small reference panel (STS), power of imputing drops considerably. In many situations, power is worse than for the best other genotyped SNP of the region. When a full sequence marker panel is available, at least the MC approach always outperforms the best other SNP. Besides that, we note that now also the pre-test with a $g p s$ threshold of 0.80 is strongly less powerful than the pre-tests with more liberal thresholds. This can be explained as follows: since the haplotype distribution of the STS is less diverse, a higher portion of individuals cannot be imputed. Thus, at a 0.80 threshold too many individuals are excluded from analysis, leaving an under-powered sample. In conclusion, the results for the STS show that a large training sample is necessary.

\section{Multiple Comparison Adjustment for Genome-Wide Power Levels}

The power-related values of table 4 have some shortcomings. In real data analysis, the disease SNPs might be in the marker panel or not, with a probability depending on the SNP coverage. Furthermore, one will try to impute all SNPs of a marker window. Thirdly, it is actually possible that a neighbouring SNP rather than the causal SNP itself will yield the best $p$ value. Therefore, we tried to evaluate the performance of $1 \mathrm{M}$ SNP panels with and without imputed SNPs in terms of genome-wide power levels. Bonferroni correction by the number of tests conducted would not be adequate, as markers are dependent due to LD. Moreover, imputed genotypes are by construction particularly highly correlated with genotyped SNPs. A Bonferroni correction would therefore underestimate the power gain obtained with imputed genotypes. The MC simulation approach computation of corrected p values could not be applied, because of the huge number of permutation replicates that would have been necessary to determine those $p$ values. To achieve the goal of computing genome-wide power values, we used an approximate technique which we will outline now. We emphasize that our approach is approximate and guided by feasibility and that we do not attempt precise genome-wide power estimation.

First, we assume that the genome can be divided into LD regions (marker windows) between which only moderate LD exist. Within each marker window, we determined $p$ values for all SNPs (imputed or not) and determined the best uncorrected $\mathrm{p}$ value $\min P$. We tried to determine empirically the efficient number of independent tests from the simulations under the null hypothesis. We computed an adjustment factor $f$ such that for $f \times$ $\min P$ the empirical level coincided with a nominal level of $\alpha=0.05$. Note that $f$ is expected to be smaller than the number of SNPs per window since the tests are correlated. We also note that $f$ is computed as an average over different windows, although $f$ will actually vary across window. For reasons of feasibility we ignored this issue and we again emphasize that the genome-wide power values shall be regarded as rough estimates. Finally, we used $f$ to determine the empirical number of independent tests in a GWAS by multiplying $f$ with the number of windows in the whole genome. These effective numbers can be found in table 5 . These numbers shall not serve as guideline for real data analysis, but they allow comparison of the different strategies considered within our power study. For real data analysis, the values of table 5 would have to be determined based on a huge number of simulated data sets and for different $\alpha$-levels. The effective numbers we find are plausible for several reasons. Firstly, the effective number of tests we compute for the full sequence panel $(1,310,000)$ comes close to the number of $1,000,000$ independent regions in the human genome 
Table 5. Multiple testing adjustment factors

\begin{tabular}{lllr}
\hline Marker panel & Imputation & $\begin{array}{l}\text { Number } \\
\text { of tests }\end{array}$ & $\begin{array}{l}\text { Effective num- } \\
\text { ber of tests }\end{array}$ \\
\hline 1M_RANDOM & none & $1,000,000$ & 600,000 \\
& HM $^{\mathrm{a}}$ & $2,600,000$ & 950,000 \\
& FS $^{\mathrm{b}}$ & $4,000,000$ & $1,130,000$ \\
\hline 1M_TAG & none & $1,000,000$ & 820,000 \\
& HM & $2,600,000$ & 980,000 \\
& FS & $4,000,000$ & $1,210,000$ \\
\hline HapMap & none & $2,600,000$ & $1,020,000$ \\
\hline Full sequence & none & $4,000,000$ & $1,310,000$
\end{tabular}

a Imputation of HapMap data; ${ }^{b}$ imputation of full sequence

postulated in a recent publication [22]. Secondly, effective numbers grow with marker panel size, but are throughout considerably lower than the respective absolute numbers. Thirdly, the effective number of tests without imputing for the 1M_RANDOM panel $(600,000)$ is lower than that for the 1M_TAG panel $(820,000)$. This is expected, since the markers of the 1M_TAG panel is chosen such that it is non-redundant. Hence, the respective single-marker tests are more independent than those of the 1M_RANDOM panel. Next, when imputed genotypes are considered, the effective number of tests is lower than the effective number of tests for the respective genotyped marker panels. Finally, comparison of the effective number of tests (including imputed SNPs) between 1M_RANDOM panel and 1M_TAG panel shows that the latter is higher (for both reference panels), which reflects the selection of 1M_TAG SNPs based on $r^{2}$ values with SNPs outside the panel. In summary, we conclude that the correction factors of table 5 are plausible and allow for approximate power comparisons.

\section{Genome-Wide Power Levels}

Within each window, we determined the best $\mathrm{p}$ value $\min P$ in two ways, either allowing $\mathrm{p}$ values of imputed genotypes or not. We multiplied $\min P$ with the effective number of tests (table 5) to be able to compute $p$ values that are corrected on a genome-wide scale. Respective power values can be found in table 6 . First, we observe that the overall power of $77.6 \%$ for full sequence data does not differ significantly from the value of $80 \%$ we envisaged when we planned the simulation set up. Considerably lower power values are obtained both for the $1 \mathrm{M}_{-}$
Table 6. Approximate genome-wide power values

\begin{tabular}{|c|c|c|c|c|}
\hline Marker panel & Imputation & $\begin{array}{l}g p s \\
\text { threshold }\end{array}$ & $\begin{array}{l}\text { ITS- } \\
\text { power }^{\mathrm{a}}\end{array}$ & $\begin{array}{l}\text { STS- } \\
\text { power }^{b}\end{array}$ \\
\hline \multirow[t]{9}{*}{ 1M_RANDOM } & none & - & 0.496 & 0.496 \\
\hline & $\mathrm{HM}^{\mathrm{c}}$ & 0.00 & 0.506 & 0.489 \\
\hline & & 0.50 & 0.513 & 0.494 \\
\hline & & 0.80 & 0.516 & 0.490 \\
\hline & & 1.00 & 0.506 & 0.468 \\
\hline & $\mathrm{FS}^{\mathrm{d}}$ & 0.00 & 0.564 & 0.508 \\
\hline & & 0.50 & 0.582 & 0.528 \\
\hline & & 0.80 & 0.577 & 0.513 \\
\hline & & 1.00 & 0.552 & 0.512 \\
\hline \multirow[t]{9}{*}{$1 \mathrm{M} \_\mathrm{TAG}$} & none & - & 0.516 & 0.516 \\
\hline & $\mathrm{HM}$ & 0.00 & 0.582 & 0.556 \\
\hline & & 0.50 & 0.591 & 0.571 \\
\hline & & 0.80 & 0.589 & 0.561 \\
\hline & & 1.00 & 0.582 & 0.533 \\
\hline & FS & 0.00 & 0.613 & 0.555 \\
\hline & & 0.50 & 0.620 & 0.582 \\
\hline & & 0.80 & 0.630 & 0.562 \\
\hline & & 1.00 & 0.598 & 0.545 \\
\hline HapMap & none & - & 0.684 & 0.684 \\
\hline Full sequence & none & - & 0.776 & 0.776 \\
\hline
\end{tabular}

a Power for ideal training sample; ${ }^{b}$ power for small training sample; ${ }^{c}$ imputation of HapMap data; ${ }^{d}$ imputation of full sequence data.

RANDOM (49.6\%) and the 1M_TAG (51.6\%) panel. Thus, even $1 \mathrm{M}$ marker panels do not provide full coverage of the genome in terms of power. Recall, however, that a considerable portion of windows that do not yield genome-wide significance will provide $\mathrm{p}$ values small enough for suggesting replication attempts (table 4). With a theoretical marker panel containing all HapMap SNPs $(2,600,000$ random SNPs) genome-wide power would still drop to $68.4 \%$.

As expected, power for the $1 \mathrm{M} \_\mathrm{TAG}$ panel is higher than that for the 1M_RANDOM marker panel. The better LD coverage of the 1M_TAG panel actually translates into improved power, although the power gain we observe in our study is small. We note that a reason for this might be that we choose a fixed number of tagging markers within each window. Thus, we will have redundant SNPs in some windows and shortage in terms of $r^{2}$ coverage in other windows. Therefore, non-window-based determined tagging panels might perform somewhat better. 
Table 7. GWA Results Male Pattern Baldness [23]

\begin{tabular}{lll}
\hline rs number & $r^{2}$ & $\mathrm{p} \mathrm{value}^{\mathrm{a}}$ \\
\hline rs2424407 & 0.034 & 0.040 \\
rs6113355 & 0.000 & 1.000 \\
rs6137432 & 0.026 & 0.514 \\
rs2208308 & 0.092 & 0.039 \\
rs2876622 & 0.540 & 0.008 \\
rs6113378 & 0.095 & 0.150 \\
rs6047633 & 0.307 & $3.0 \times 10^{-4}$ \\
rs873137 & 0.131 & 0.201 \\
rs6047637 & 0.376 & 0.0013 \\
rs6137444 & 0.046 & 0.392 \\
rs6047641 & 0.136 & 0.006 \\
rs6047646 & 0.097 & 0.548 \\
rs17665823 & 0.058 & 0.033 \\
rs12624315 & 0.047 & 0.307 \\
rs2180439 & & $3.9 \times 10^{-7}$ \\
\hline
\end{tabular}

${ }^{\mathrm{a}} \mathrm{r}^{2}$ with $\mathrm{rs} 2180439 ;{ }^{\mathrm{b}} \mathrm{p}$ value obtained with Armitage's Trend Test and real genotype data.

When imputed genotypes are included into association analysis, power always improves substantially. Again, we first discuss the results for the ITS. In contrast to table 4, we now observe a power gain also for the 1M_RANDOM panel with HapMap reference panel. This can be explained by the fact that now multiple SNPs are imputed.

In concordance with table 4 , there is no clear trend for the power values corresponding to the various $g p s$ threshold, despite that the power for the 1.00 threshold is reduced. Therefore, we discuss only the power levels belonging to the threshold of 0.00 further. For both marker panels, a power gain is observed when imputation on the HapMap scale is considered. Power increases from 49.6 to $50.6 \%$ for the $1 \mathrm{M} \_$RANDOM and from 51.6 to $58.2 \%$ for the 1M_TAG panel. Further power gain can be observed when we consider imputing on a full sequence panel. The power gains for both the 1M_RANDOM panel (improvement to 56.4\%) and the 1M_TAG (improvement to $61.3 \%$ ) are substantial, highlighting the potential of a denser reference panel. Thus, enlarging the marker density of the HapMap would aid association analysis of imputed data. This is particularly true for marker panels that are not subsets of the HapMap panel.

Genome-wide power levels for the STS panel are generally lower. The difference between ITS and STS is not as striking as in table 4 , since we now consider adjusted $p$ values, based on all imputed and non-imputed SNPs of the region. As for the point-wise power values, we observe reduced power for the $g p s$ thresholds 0.80 and 1.00. At the 0.50 threshold we still observe a power gain with imputing. However, it becomes obvious that the gain is not substantial, because of the small sample size of the training sample.

\section{Application to Real Data Set}

Hillmer et al. [23] recently identified a new locus for male pattern baldness on chromosome 20p11. In an initial GWAS (297 cases, 347 controls), the strongest association within the region was found for SNP rs2180349 $\left(\mathrm{p}=3.9 \times 10^{-7}\right.$, table 7$)$. The SNP was confirmed in an independent replication sample (combined $\mathrm{p}=2.7 \times$ $10^{-15}$ ). Here, we address the following question: would it have been possible to detect the SNP with imputed genotypes? In order to avoid trivial imputation, we selected those flanking SNPs that showed pairwise $r^{2}$ values with rs2180349 smaller than 0.90 and an association $p$ value worse than $1 \times 10^{-4}$. This resulted in a list of 14 SNP markers. Their $r^{2}$ values with rs2180349 and single-marker association results can be found in table 7. For evaluation of our imputation methods, we masked SNP rs2180349 in the GWAS data. Results from the association analysis of the imputed SNP rs2180349 can be found in table 8.

We first consider the results for the ideal training sample (ITS). The ITS data was obtained by simply doubling the GWAS sample and genotype data and setting the affection status to unknown for the double data set. With the MC approach, a strong association signal is obtained $\left(p=1.7 \times 10^{-6}\right)$ that clearly outperforms the best $p$ value of the genotyped markers $\left(\mathrm{p}=3.0 \times 10^{-4}\right.$, table 7$)$.

The minor allele frequency for rs 2180493 was 0.39 , resulting in a priori genotype probabilities of $0.156,0.478$ and 0.366 , respectively. After imputation, many individuals had genotype probability vectors $V_{1}=(0.137,0.789$, $0.074)$ or $V_{2}=(0.834,0.159,0.008)$ of the form $V=\left(f_{11}, f_{12}\right.$, $\left.f_{22}\right)$. For both $V_{1}$ and $V_{2}$, the most likely genotype has a probability of about $80 \%$. However, the $g p s$ value for $V_{1}$ is 0.484 and the value for $V_{2}$ is 0.822 . $V_{2}$ contains indeed more information: although the genotype $(1,1)$ has the lowest prior, its probability after imputation is $83.4 \%$. In addition, the possibility that the genotype is $(2,2)$ can almost be excluded $\left(f_{22}=0.008\right)$. Thus, $V_{2}$ is more informative and individuals with $V_{2}$ are kept when a gps threshold of 0.80 is applied while individuals with $V_{1}$ are removed already at a threshold of 0.50 .

Consistent with our simulation study, the $\mathrm{p}$ values of the pre-test for rs2180349 are generally worse than with 
Table 8. p values for imputed SNP rs2180439 for three different training samples

\begin{tabular}{|c|c|c|c|c|c|c|}
\hline \multirow[t]{2}{*}{ gps score } & \multicolumn{2}{|c|}{ HapMap parents ${ }^{\mathrm{a}}$} & \multicolumn{2}{|c|}{ HapMap trios ${ }^{\mathrm{b}}$} & \multicolumn{2}{|c|}{ Ideal training sample } \\
\hline & portion $^{c}$ & $\mathrm{p}$ value & portion & $\mathrm{p}$ value & portion & $\mathrm{p}$ value \\
\hline 0.00 & 1.000 & $3.4 \times 10^{-4}$ & 1.000 & $4.3 \times 10^{-4}$ & 1.000 & $2.9 \times 10^{-4}$ \\
\hline 0.50 & 0.932 & $3.0 \times 10^{-4}$ & 0.754 & $1.9 \times 10^{-3}$ & 0.957 & $2.7 \times 10^{-4}$ \\
\hline 0.55 & 0.650 & $4.3 \times 10^{-6}$ & 0.668 & $2.5 \times 10^{-6}$ & 0.944 & $2.8 \times 10^{-4}$ \\
\hline 0.60 & 0.551 & $5.0 \times 10^{-6}$ & 0.550 & $1.0 \times 10^{-5}$ & 0.683 & $4.2 \times 10^{-6}$ \\
\hline 0.80 & 0.215 & $2.4 \times 10^{-2}$ & 0.233 & $1.8 \times 10^{-2}$ & 0.300 & $4.7 \times 10^{-3}$ \\
\hline 1.00 & 0.012 & $7.9 \times 10^{-1}$ & 0.007 & $3.0 \times 10^{-1}$ & 0.016 & $5.8 \times 10^{-1}$ \\
\hline $\mathrm{MC}^{\mathrm{d}}$ & 1.000 & $2.8 \times 10^{-6}$ & 1.000 & $2.3 \times 10^{-6}$ & 1.000 & $1.7 \times 10^{-6}$ \\
\hline
\end{tabular}

${ }^{a}$ HapMap data is used as training data, but child information is ignored; ${ }^{b}$ HapMap data is used as training data and child information is used; ${ }^{c}$ portion of data that is kept after removing individuals that have a gps score below the threshold; ${ }^{d}$ values for Monte-Carlo method.

the MC approach. With a gps threshold of 0.80 only $30 \%$ of the data is kept and the association signal drops to $\mathrm{p}=$ $4.7 \times 10^{-3}$. With a $g p s$ threshold of 1.00 only $1.6 \%$ of the data is kept, leaving no signal. The best pre-test $p$ value $\mathrm{p}=4.2 \times 10^{-6}$ is obtained with a $g p$ s threshold of 0.60 , a value that was not considered in our simulation study. In general, the pre-test $\mathrm{p}$ values are strongly affected by the choice of the gps threshold.

Using HapMap trios for training performed surprisingly well. The MC $p$ value $p=2.3 \times 10^{-6}$ was almost as good as with the ITS. Again, we observe high variability of the $p$ value obtained with the pre-test, depending on the choice of the $g p s$ threshold.

Finally, we tried to evaluate the benefit of using child information in haplotyping. To this purpose, we only used the HapMap parents in the training sample. MC $\mathrm{p}$ value and the best pre-test $p$ value were slightly worse than for the full HapMap trios. Thus, child information is useful also in our real data set, but we were not able to detect a particularly strong effect.

In summary, we obtained very good results with the imputation methods in our real data analysis. We also checked their performance with less prediction markers. However, when we used only the three SNPs with the highest $r^{2}$ with rs2180439, we were not able to obtain an association signal better than $\mathrm{p}=4 \times 10^{-4}$ in any of the constellations. Thus, it is actually necessary to use many SNPs for prediction also for our real data set. The real data also confirms that a gps threshold around 0.50 is a good choice for the pre-test, but the exact cut-off is obviously hard to define. Thus, the MC approach should be regarded as the method of choice.

Association Analysis with EM-Imputed Genotypes

\section{Discussion}

We have introduced a MC simulation procedure for association testing of imputed SNPs that is robust with respect to non-matching $\mathrm{LD}$ patterns of training and study sample. In addition, we have introduced a pre-test that allows quick computation of an approximate upper bound of the true $\mathrm{p}$ value. In practice, the two approaches can be combined and be supplemented with a 'pre-pretest'. First, in case not enough computer running time is available, pre-selected $L D$ regions can be identified by the presence of at least one genotyped SNP in the LD region that is nominally significant at a liberal level. Second, imputed SNPs are tested for association with the pre-test. Third, the top-ranking pre-test $\mathrm{p}$ values are validated with the MC approach. We have to emphasize that our current implementation in FAMHAP does not provide those necessary features for application to a GWAS. The strategy just described has to be implemented by the user group itself, which is clearly a demanding task. However, usage in gene-based analysis and using the MC approach for validation is supported by our software and doable for all users. We actually believe that validation with the MC approach is the most important feature our implementation can offer. Indeed, our simulation study and real data analysis has shown that the choice of an appropriate $g p s$ cut-off for exclusion of individuals is difficult.

Our simulation study shows that imputing can substantially increase power of genome-wide single-marker analysis. However, necessary prerequisites are that, first, the training sample ethnically matches the study sample, and, second, that the training sample is large enough to 
capture the full haplotype diversity of the study sample. Third, a dense reference marker panel, ideally full sequence data, leads to further increase in power. We conclude that it would be ideal if every country (ethnic group) had its own, large training sample (more than 1000 individuals) with full sequence data. It seems that this is a realistic goal for the future.

We make explicit that our conclusions are based on the assumption that the available study sample size is limited. In particular, we did not calculate the efficiency of imputing in terms of genotyping effort. The later has been done in a recent publication by Anderson et al. [24]. They conclude that when sample size is not limited, rather small study marker panels (300K) are cost efficient.

We finally note that our power values under imputing are still lower than the power that would be obtained with a fully genotyped, complete marker panel. Hence, from the point of view of limited sample size, full sequence data is desirable for GWAS. These are bad and good news at the same time. The good news are that it is likely that additional variants can be found with full sequence panels in the very same samples that already lead to positive association findings using SNP panels with 100,000 or 500,000 SNPs.

\section{Acknowledgments}

The work was supported by grant BE 3828/3-1 from the Deutsche Forschungsgemeinschaft.

\section{Electronic Database Information}

URLs for data presented herein are as follows: FAMHAP: http://famhap.meb.uni-bonn.de

\section{References}

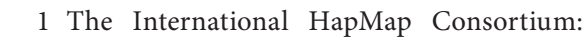
The International HapMap Project. Nature 2003;426:789-796.

2 Evans DM, Cardon LR, Morris AP: Genotype prediction using a dense map of SNPs Genet Epidemiol 2004;27:375-384.

-3 de Bakker PIW, Yelensky R, Pe'er I, Gabriel SB, Daly MJ: Efficieny and power in genetic association studies. Nat Genet 2005;37:12171223.

$\checkmark 4$ Nicolae DL: Testing untyped alleles (TUNA)-applications to genome wide association studies. Genet Epidemiol 2006;30: 718-727.

5 Marchini J, Howie B, Myers S, McVean G, Donnelly P: A new multipoint method for genome-wide association studies by imputation of genotypes. Nat Genet 2007;39:906913.

-6 Browning SR, Browning BL: Rapid and accurate haplotype phasing and missing-data inference for whole-genome association studies by use of localized haplotype clustering. Am J Hum Genet 2007;81:1084-1097.

-7 BurdickJT, Chen WM, Abecasis GR, Cheung VG: In silico method for inferring genotypes in pedigrees. Nat Genet 2006;38:1002-1004.

$\checkmark 8$ Zaitlen N, Kang HM, Eskin E, Halperin E: Leveraging the HapMap correlation structure in association studies. Am J Hum Genet 2007;80:683-691

-9 Excoffier L, Slatkin M: Maximum-likelihood estimation of molecular haplotype frequencies in a diploid population. Mol Biol Evol 1995;12:921-927.
10 Hawley ME, Kidd KK: HAPLO: a program using the EM algorithm to estimate the frequencies of multi-site haplotypes. Hum Hered 1995;86:409-411.

11 Long JC, Williams RC, Urbanek M: An E-M algorithm and testing strategy for multi-locus haplotypes. Am J Hum Genet 1995;56: 799-810.

12 Qin ZS, Niu T, Liu JS: Partition-ligation-expectation-maximization algorithm for haplotype inference with single-nucleotide polymorphisms. Am J Hum Genet 2002;71: 1242-1247.

13 Eronen L, Geerts F, Toivonen H: HaploRec: efficient and accurate large-scale reconstruction of haplotypes. BMC Bioinfromatics 2006;7:542.

14 Stephens M, Smith NJ, Donnelly P: A new statistical method for haplotype reconstruction from population data. Am J Hum Genet 2001;68:978-989.

15 Scheet P, Stephens M: A fast and flexible statistical model for large-scale population genotype data: applications to inferring missing genotypes and haplotypic phase. Am J Hum Genet 2006;78:629-644.

16 Marchini J, et al: A comparison of phasing algorithms for trios and unrelated individuals. Am J Hum Genet 2006;78:437-450.

17 Becker T, Knapp M: Maximum-likelihood estimation of haplotype frequencies in nuclear families. Genet Epidemiol 2004;27:2132.

18 Becker T, Knapp M: Efficiency of haplotype frequency estimation when nuclear family information is included. Hum Hered 2002; 54:45-53.
19 Schaid DJ: Relative efficiency of ambiguous vs. directly measured haplotype frequencies. Genet Epidemiol 2002;23:426-443.

20 Becker T, Cichon S, Joenson E, Knapp M: Multiple testing in the context of haplotype analysis revisited: application to case-control data. Ann Hum Genet 2005;69:747756.

21 The International HapMap Consortium: A second generation human haplotype map of over 3.1 milllion SNPs. Nature 2007;449: 851-862.

-22 The Wellcome Trust Case Control Consortium: Genome-wide association study of 14,000 cases and seven common diseases and 3,000 shared controls. Nature 2007;447: 661-683.

23 Hillmer AM, Brockschmidt FF, Hanneken S, Eigelshoven S, Steffens M, Flaquer A, Herms S, Becker T, Kortuem AK, Nyholt DR, Zhao ZZ, Montgomery GW, Martin HG, Muehleisen TW, Alblas MA, Möbus S, Jöckel KH, Bröcker-Preuss M, Erbel R, Reinartz R, Betz RC, Cichon S, Propping P, Baur MP, Wienker TF, Kruse R, Nöthen MM: A genomewide association scan identifies new susceptibility variants for male pattern baldness on chromosome 20p11. Nat Genet 2008;40: 1279-1281.

24 Anderson CA, Petterson FH, Barrett JC, Zhuang JJ, Ragoussis J, Cardon LR, Morris AP: Evaluating the effects of imputation on the power, coverage, and cost efficiency of genome-wide SNP platforms. Am J Hum Genet 2008;83:1-8 